\title{
Prognostic value of changes in the expression of stem cell markers in the peripheral blood of patients with colon cancer
}

\author{
MARIA-ELENA PADÍN-IRUEGAS ${ }^{1}$, MICHEL HERRANZ-CARNERO ${ }^{2}$, SANTIAGO AGUIN-LOSADA $^{1}$, \\ ELENA BROZOS-VAZQUEZ ${ }^{1}$, U. ANIDO-HERRANZ ${ }^{1}$, JOSE-RAMON ANTUNEZ-LOPEZ ${ }^{3}$, \\ ALVARO RUIBAL-MORELL ${ }^{4}$ and RAFAEL LÓPEZ-LÓPEZ ${ }^{1}$ \\ ${ }^{1}$ Department of Medical Oncology, ${ }^{2}$ Molecular Oncology and Imaging Program, Molecular Imaging Group, \\ IDIS, Departments of ${ }^{3}$ Pathology, ${ }^{4}$ Nuclear Medicine, Faculty of Medicine, \\ University Hospital of Santiago de Compostela, La Coruña, Galicia, Spain
}

Received January 4, 2013; Accepted February 18, 2013

DOI: $10.3892 /$ or.2013.2368

\begin{abstract}
Cancer stem cells play an important role in carcinogenesis and resistance to treatment and may lead to metastasis. The isolation of circulating stem cells involves cell sorting based on the presence of cell surface markers. Many surface markers such as CD133, c-Kit, SOX, OCT4 and TWIST have been reported. In the present study, we determined the expression of different stem cell markers and their variation in expression at different stages of the treatment process. Samples of EDTA blood were collected from metastatic colorectal cancer patients, and circulating cancer stem cells were isolated for the analysis of the expression of stem cell markers using RT-PCR. These findings were correlated with the response to therapy. All statistical analyses were performed using the GraphPad Prism 5.03 software. Significant differences were found in the expression levels of the markers CD133, SOX2, OCT4 and TWIST1. No differences were found in c-Kit expression. Correlation in the expression levels of most of the markers was observed. Expression of CD133, OCT4, SOX2 and TWIST1 had a predictive value for colon cancer behavior. Evaluation of this stem cell gene expression panel may be useful for predicting the response during the process of treatment, and the relative easy access to samples facilitates this method. Moreover the correlation between CD133 and TWIST1 expression may be associated with tumor regrowth and metastatic relapse.
\end{abstract}

\section{Introduction}

Tumors derived from epithelial tissues as lung, prostate, colon or breast are the most prevalent in industrialized countries.

Correspondence to: Dr Maria-Elena Padín-Iruegas, Laboratory of Translational Oncology, Department of Clinical Oncology, University Hospital of Santiago de Compostela, Travesia Choupana s/n, Santiago de Compostela 15706, La Coruña, Galicia, Spain

E-mail: mariaelena.padin.iruegas@sergas.es; mepadin@vigo.es

Key words: cancer stem cells, colon cancer, CD133, OCT4, SOX2, TWIST1
Colorectal cancer is one of the most common causes of cancerrelated mortality worldwide. For colorectal cancer, surgical resection of the tumor is commonly the first therapeutic action with curative intent. However, $50 \%$ of patients develop metastatic disease which is incurable with current treatments. Although the median overall survival has increased as a result of the improvement in systemic therapies such as new chemotherapy agents and monoclonal antibodies, the median overall survival has reached a plateau at 24 months. Therefore, one of the main challenges in colorectal cancer is to develop new strategies to inhibit disease progression $(1,2)$.

The stem cell hypothesis postulates that cancers, as they occur in normal tissues, consist of at least two types of cell populations: cancer stem cells (CSCs) and adult stem cells. CSCs exhibit all of the characteristics of stem cells: the capability of self-renewal, unlimited proliferation potential, multi-line differentiation and the capability to form new cancer stem cells and adult cancer stem cells through asymmetric division. It has been hypothesized that CSCs play a role in tumor chemoresistance. They have also been termed cancer initiating cells as they retain the ability to form colonies, and are also responsible for tumor recurrence and refractoriness (3-9). It has been suggested that colorectal cancer is caused by an alteration in the normal homeostasis of stem cells (Fig. 1).

The standard procedure for isolating circulating stem cells involves cell sorting of the cell subpopulation based on the presence of cell surface markers. Many of these surface markers have been reported to be present in CSCs. The expression of these markers change depending on the organ that is consider. One of the putative markers highly specific in colon cancer is CD133 (also known as prominin-1). The CD133 gene is located on chromosome 4p15.32 and encodes a cell surface glycoprotein consisting of 5 transmembrane domains and 2 large glycosylated extracellular loops. Yet, the function of CD133 has not been established to date. An association between levels of CD133 and the prognosis of colorectal cancer patients have been established. Several authors have reported that high expression of CD133 is associated with poor prognosis and distant metastasis, while another study indicates it is an independent factor. Initially identified in hematopoietic stem cells, CD133 is a shared cancer stem cell marker in multiple solid 


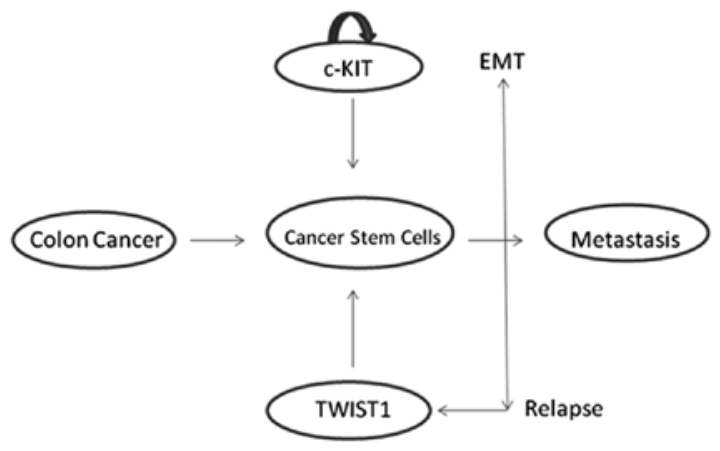

Figure 1. Scheme of the theoretical behavior of stem cells in cancer, their maintenance and transformation to produce distant metastases.

tumors including brain, breast, lung, liver, prostate, pancreas, medulloblastoma and melanoma (10-24).

c-Kit, also called KIT, CD117 or c-Kit receptor, is a cytokine receptor expressed on the surface of several types of stem cells. c-Kit was first identified as the cellular homolog of the feline sarcoma viral oncogene V-kit. Altered forms of this receptor may be associated with certain types of cancer. This protein is a type 3 transmembrane receptor for mast cell growth factor, also known as stem cell factor (MGF). The kit gene encodes the human homolog of the proto-oncogene c-Kit. Mutations in this gene are associated with gastrointestinal stromal tumors, testicular seminoma, mast cell disease, melanoma, acute myeloid leukemia, and benign disease such as piebaldism. Multiple transcript variants encoding different isoforms have been found for this gene (25).

OCT4 is a commonly used synonym for POU5F1 (POU class 5 homeobox 1). It is a homeodomain transcription factor of the POU family. This protein is critically involved in the self-renewal of undifferentiated embryonic stem cells; thus, it is commonly used as a marker of undifferentiated cells. OCT4 expression must be strictly regulated; excess or default causes cell differentiation. The OCT4 transcription factor is initially active as a maternal factor in the oocyte but remains active in embryos throughout the preimplantation period. OCT4 expression is associated with the undifferentiated phenotype and tumors. OCT4 can form a heterodimer with SOX2; thus, these two proteins bind DNA together $(26,27)$.

OCT4 and SOX2 transcription factors are essential for normal pluripotent cell development and maintenance. They are also known as reprogramming genes, inducing an embryonic stem cell-like state. SOX2 plays a critical role in cell fate determination, differentiation and proliferation; is involved in cancer events such as invasion and metastasis, and plays a role in conferring a low differentiation phenotype in tumors. TWIST1, a master regulator of embryonic morphogenesis, plays an essential role in metastasis. Ectopic expression of TWIST1 results in loss of E-cadherin-mediated cell-cell adhesion, activation of mesenchymal markers, and induction of cell motility, suggesting TWIST contributes to metastasis by promoting an epithelial-mesenchymal transition (EMT) (28-30).

For epithelial tumors, EMT is consider to be a crucial event in the metastatic process, which involves the disruption of epithelial cell homeostasis and the acquisition of a migratory mesenchymal phenotype allowing cells to travel to the site of metastasis formation without being affected by conventional treatment. Assuming metastasis requires dissemination of tumor stem cells showing EMT, it seems likely that this type of cell is detectable among circulating tumor cells found in cancer patients (31).

The objective of our study was to determine the expression of markers of CSCs in circulating tumor cells (CTCs) in the peripheral blood of colon cancer patients, and assess the pathway expression at various time points during treatment in respect to the response of the disease to chemotherapy.

\section{Materials and methods}

We enrolled 29 metastatic colorectal cancer patients. Eligibility patient criteria were as follows: age $\geq 18$ years of age, clinical stage IV based on the International TNM classification (40), performance status of $0-2$, and the presence of no other malignancies. All of the patients were treated with fluoropyrimides. A second group of 16 healthy volunteers, matched for age, were used as the control group. All patients and volunteers signed informed consent forms. This study was approved by the Ethics Committee for Clinical Research of Galicia, Spain (CEIC Galicia: 2010/238).

Samples of EDTA blood $(10 \mathrm{ml})$ were collected for detection of circulating CSC markers. Blood samples were evaluated for expression of c-Kit, CD133, SOX2, OCT4 and TWIST1 before and during chemotherapy.

In the patient group, samples were collected at 3 different time points during therapy: prior to chemotherapy; before the second cycle of treatment, and finally before the fifth cycle of treatment. The patient response was evaluated following the Response Evaluation Criteria In Solid Tumors (RECIST 1.1) (39) and was confirmed by computed tomography (CT) according to 4 categories: complete response (CR), partial response (PR), stable disease (ED) and progressive disease (PD).

Samples were centrifuged at $2,000 \mathrm{x} \mathrm{g}$, for $10 \mathrm{~min}$; the serum phase was separated and frozen at $-80^{\circ} \mathrm{C}$. Buffy coat was collected and processed with lysis (ammonium chloride, Tris, $\mathrm{ddH}_{2} \mathrm{O}$ ) and washed with PBS. The dry pellet was maintained at $-80^{\circ} \mathrm{C}$ until RNA isolation. RNA was purified using the QIAamp RNA Blood Mini kit (Qiagen Inc., USA), according to the manufacturer's instructions. Complementary DNA (cDNA) was synthesized with random hexamer primers (Deoxynucleoside Triphosphate Set; Roche, Germany) with $10 \mathrm{mM} \mathrm{MgCl}{ }_{2}, \mathrm{MuLV}$ reverse transcriptase, PCR buffer, RNAse inhibitor and random hexamers (Applied Biosystems, USA). The resulting cDNA was stored at $-20^{\circ} \mathrm{C}$ until further use.

Quantitative RT-PCR analysis was carried out with SYBR-Green Master Mix (Applied Biosystems), using Applied Biosystems 7500 Real-Time PCR system according to the manufacturer's instructions. Primers for c-Kit and GAPDH were designed with Vector NTI Advance ${ }^{\mathrm{TM}} 11$ (Invitrogen), and primers for CD133, SOX2, OCT4 and TWIST1 were designed with Primer3 software (version 0.4.0; Biology Workbench) (Table I). To avoid the influence of genomic contamination, forward and reverse primers for each gene were located in different exons. PCR was performed using a final volume of $20 \mu \mathrm{l}$ with SYBR-Green PCR Master Mix, using $1 \mu \mathrm{l}$ cDNA. 
Table I. Sequences of the specific primers designed to perform RT-PCR for the stem cell markers.

Primer sequences

\begin{tabular}{lll}
\hline c-Kit & $\begin{array}{l}\text { Sense } \\
\text { Antisense }\end{array}$ & 5'-GATGGCACCTGAAAGCATTT-3' \\
& 5'TCGAAGCCTTCCTTGATC-3' \\
GAPDH & Sense & 5'-AGGTCATCCATGACAACTTTG-3' \\
& Antisense & 5'-TTCAGCTCAGGGATGACCTT-3' \\
CD133 & Sense & 5'-TTTCCAAATCCAGGAGCAAC-3' \\
& Antisense & 5'-ACAGGAAGGGAGGGAGTCAT-3' \\
SOX2 & Sense & 5'-ACCAGCGCATGGACAGTTAC-3' \\
& Antisense & 5'-TCATGTAGGTCTGCGAGCTG-3' \\
OCT4 & Sense & 5'-AGTGAGAGGCAACCTGGAGA-3' \\
& Antisense & 5'-ACACTCGGACCACATCCTTC-3' \\
TWIST1 & Sense & 5'-ACTGGCCTGCAAAACCATAG-3' \\
& Antisense & 5'-TGCATTTTACCATGGGTCCT-3' \\
\hline
\end{tabular}

Cycling conditions consisted of $95^{\circ} \mathrm{C}$ for $10 \mathrm{~min}$, followed by 40 cycles at $95^{\circ} \mathrm{C}$ for $15 \mathrm{sec}$ and $60^{\circ} \mathrm{C}$ for 1 min each. A melting curve was then obtained.

Relative gene expression levels were determined using the quantitative curve method. Quantitative normalization of cDNA in each sample was performed using GAPDH gene expression as an internal control. Target gene messenger RNA (mRNA) levels were expressed as ratios to GAPDH mRNA levels. RT-PCR assays were carried out in duplicate for each sample, and the mean value was used for calculation of the mRNA expression levels.

Data are presented as median, range and mean \pm standard deviation. Significance between two groups was determined by an unpaired two-tailed Student's t-test. $\mathrm{P}<0.05$ was considered to indicate a statistically significant difference. Spearman's correlation coefficient non-parametric measure of statistical dependence between two variables was used; a perfect Spearman correlation of +1 or -1 occurs when each of the variables is a perfect monotone function of the other. All statistical analyses were carried out using GraphPad Prism 5.03 software.

\section{Results}

Patient characteristics. We evaluated the expression of CSC markers in the peripheral blood of 29 colon cancer patients. The patients had stage IV disease and consisted of 16 males and 13 females with a median age of 63 years (range 42-85). Three patients dropped out of the study due to disease progression.

Distribution of the localization of the tumors is as follows: 6 were situated in the ascending colon, 2 were in the transverse colon, 6 were in the descending colon, 5 were in the sigmoid colon, 8 were in the rectum, and the sites was unknown for 2 patients diagnosed at other hospitals. All of the cases were adenocarcinomas: 9 well differentiated, 19 moderately differentiated and 1 poorly differentiated.

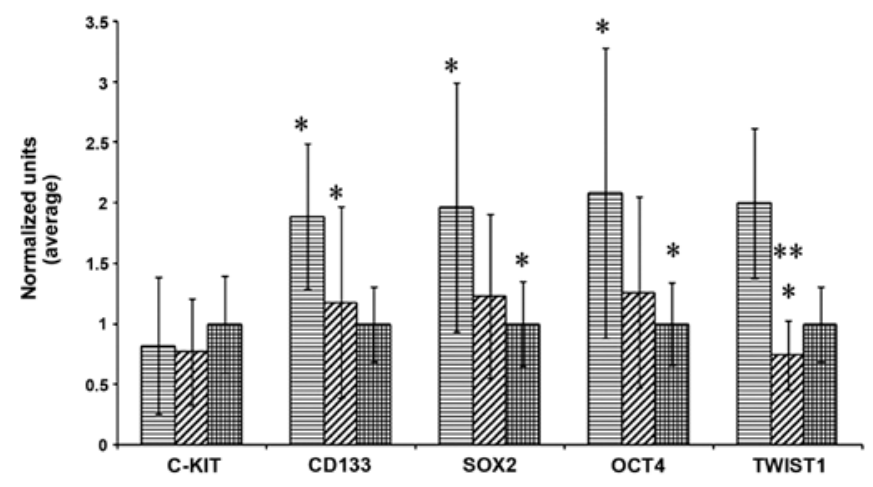

Figure 2. Expression of c-Kit, CD133, SOX2, OCT4 and TWIST1 in the DNA of cells isolated from the peripheral blood of patients prior to chemotherapy (columns with horizontal lines, left) and following the first dose of chemotherapy (columns with oblique lines, middle), compared with healthy donors (columns with squares, right). Prior to chemotherapy, apart from c-Kit, all of the markers demonstrated differences between the patients and controls; levels were higher than in the healthy donors, but they were reduced after treatment. There were no significant differences between the controls and patients who received one dose of chemotherapy. When comparing patients before and after chemotherapy, the levels of markers were significantly reduced, in particular TWIST1. Data were normalized to establish the comparison. "Indicates differences with statistical significance between patients and controls. ${ }^{* * * *}$ Indicates differences with statistical significance between patients and controls at $\mathrm{P}<0.001$.

The control group included 16 volunteers with a median age of 60 years (range 42-83).

We analyzed the CEA values at three different time points during the sample collection, but no statistical differences were noted, although a reduction in the levels of CEA was noted when patients demonstrated a response to chemotherapy.

Expression of the various markers. DNA obtained from cells isolated from the peripheral blood samples was analyzed by RT-PCR. The signal expression of stem cell markers, c-Kit, CD133, SOX2, OCT4 and TWIST1, in the patients were compared with the healthy control group.

The expression of the markers was also assessed in colon tumor tissue samples, following the same procedure to obtain DNA for RT-PCR analysis.

When we compared the patients not yet treated with chemotherapy with the controls, significant differences in the expression of CD133, SOX2, OCT4 and TWIST1 $(\mathrm{p}=0.0002$; $\mathrm{p}=0.0182 ; \mathrm{p}=0.0217 ; \mathrm{p}=0.0031$, respectively) (Fig. 2) were noted. Expression of c-Kit was not significantly different.

When we analyzed the same marker levels in the peripheral blood in the patients following administration of the first cycle of chemotherapy and prior to the administration of the second, the results obtained were similar. There were significant differences in the expression levels of the same markers: CD133 ( $\mathrm{p}=0.008), \operatorname{SOX} 2(\mathrm{p}=0.036)$, OCT4 $(\mathrm{p}=0.04)$ and TWIST1 $(\mathrm{p}=0.0002)$. No difference was found in $\mathrm{c}-\mathrm{Kit}$ expression (Fig. 2).

We next divided the patients according to their response to treatment. There were two groups: one group included patients with no change, and another group exhibited PR or CR to treatment. We found differences in the expression pattern of the markers in the patients 'in progression' compared to those 'with response'. In the patient with tumor progression, 


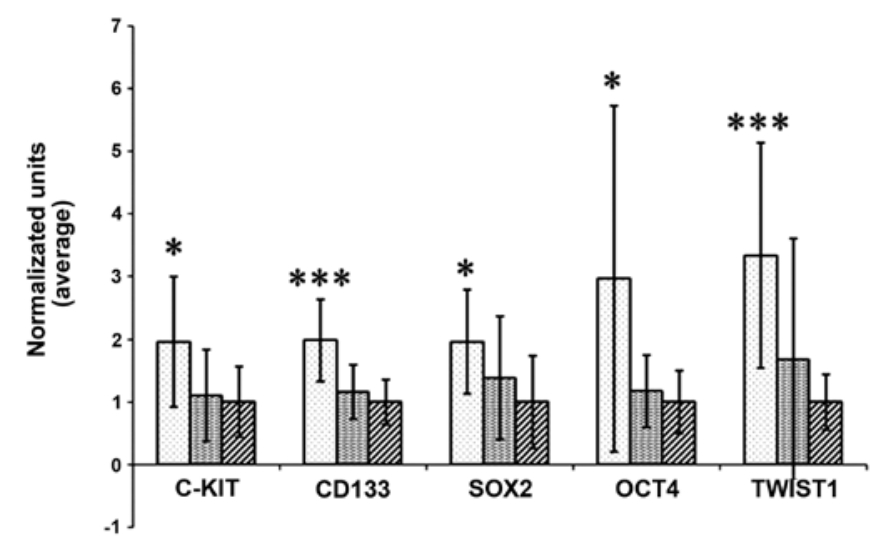

Figure 3. Expression of c-Kit, CD133, SOX2, OCT4 and TWIST1 in the DNA of cells isolated from the peripheral blood of patients prior to chemotherapy (column with dots, left) and after the fourth dose of chemotherapy (column with horizontal lines, middle), compared with healthy controls (columns with oblique lines, right). The levels of all markers were increased in patients with disease progression when compared with the controls, indicating no response to chemotherapy and tumor spread. Notable were the levels of CD133 and TWIST1, a colon stem cell marker and epithelialmesenchymal transition marker, respectively. Conversely, there were no differences between the levels of markers between patients responsive to chemotherapy and the controls. Data were normalized to establish the comparison. "Indicates differences with statistical significance between patients and controls. ${ }^{* * *}$ Indicates differences with statistical significance between patients and controls at $\mathrm{P}<0.001$.

we found differences in the levels of all markers, including $\mathrm{c}-\mathrm{Kit}$. The differences were significant: for $\mathrm{c}-\mathrm{Kit}(\mathrm{p}=0.0046)$, for CD133 ( $\mathrm{p}=0.0001)$, for SOX2 $(\mathrm{p}=0.0042)$, for OCT4 $(\mathrm{p}=0.0094)$ and for TWIST1 $(\mathrm{p}<0.001)$. In the patient group with no progression, there were no differences in expression levels when compared with the controls (Fig. 3).

In addition to the comparison between patients and controls, we compared the different groups of patients in regards to response to therapy.

When comparing patients prior to chemotherapy with patients who had tumor progression after 4 cycles of treatment, differences only in the expression of TWIST1 $(p<0.0001)$ were noted. When we compared the patients prior to treatment with those showing a response after 4 cycles of chemotherapy, differences in the levels of CD133 $(\mathrm{p}=0.0002)$ and OCT4, $(\mathrm{p}=0.0073)$ were noted.

Comparing patients following administration of 4 cycles of treatment with those who were administered only one dose, the patient group with tumor progression had high expression levels of all the markers with significant $\mathrm{P}$-values [c-Kit $(\mathrm{p}=0.0011)$, CD133 $(\mathrm{p}=0.01)$, SOX2 $(\mathrm{p}=0.02)$, OCT4 $(\mathrm{p}=0.04)$ and TWIST1 $(\mathrm{p}<0.0001)]$. In the case of the group who showed respond to therapy, there were no significant differences.

Finally, we compared the patients after the fourth cycle of chemotherapy with tumor progression and without progression; expression of all markers demonstrated significant differences excluding SOX2.

Correlation between markers. After evaluation of marker expression, we applied the Spearman's test to ascertain the correlation between them. In the patients prior to chemotherapy, we found a negative correlation between c-Kit and r:0.89

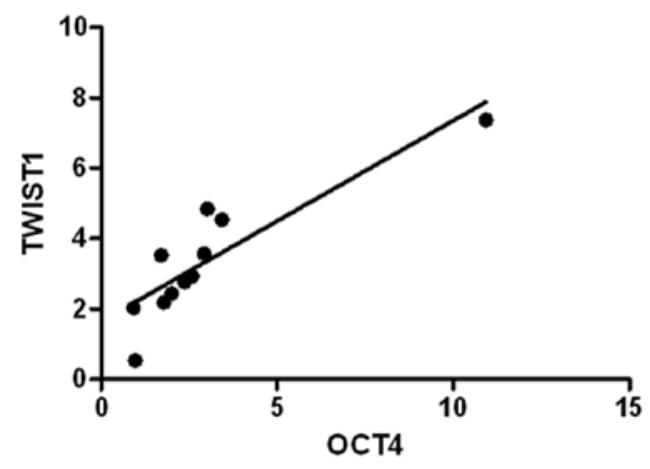

Figure 4. Correlation between levels of expression of OCT4 and TWIST1 in patients who received 4 cycles of chemotherapy. Spearman's correlation was applied to determine the grade of association between the two variables. The data obtained, 0.89, indicates a high level of association between OCT4 and TWIST1, two of the markers of immature stem cells, suggesting transformation of the cancer stem cells into a mesenchymal state, to facilitate migration and invasion into other tissues.

SOX2 $(r=0.5, p=0.03)$, while positive correlations were noted between CD133 and SOX2 ( $\mathrm{r}=0.6, \mathrm{p}=0.01), \mathrm{CD} 133$ and OCT4 $(\mathrm{r}=0.6, \mathrm{p}=0.01), \mathrm{CD} 133$ and TWIST1 $(\mathrm{r}=0.9, \mathrm{p}=0.002)$, and SOX2 and OCT4 ( $r=0.8, p=0.0003)$.

In the patients who received one dose of chemotherapy a correlation was noted between CD133 and SOX2, OCT4 and TWIST1, with the following values: CD133 and SOX2 $(r=0.55, p=0.04)$; CD133 and OCT4 $(r=0.88, p<0.0001)$, SOX2 and OCT4 $(r=0.65, p=0.01)$. Finally, there was no correlation between TWIST1 and CD133, TWIST1 and SOX2, TWIST1 and OCT4 and between c-Kit and the rest of the markers.

In the patients who received 4 cycles of chemotherapy with tumor progression, a positive correlation was noted only between OCT4 and TWIST1 ( $\mathrm{r}=0.89, \mathrm{p}=0.0002$ ) (Fig. 4). In the same patient group but without tumor progression, a significant correlation was found between c-Kit and CD133 $(\mathrm{r}=0.55, \mathrm{p}=0.001), \mathrm{c}-\mathrm{Kit}$ and OCT4 $(\mathrm{r}=0.47, \mathrm{p}=0.04), \mathrm{c}-\mathrm{Kit}$ and TWIST1 ( $\mathrm{r}=0.49, \mathrm{p}=0.03), \mathrm{CD} 133$ and OCT4 ( $\mathrm{r}=0.53, \mathrm{p}=0.02)$, CD133 and TWIST1 ( $\mathrm{r}=0.61, \mathrm{p}=0.006)$, OCT4 and TWIST1 $(\mathrm{r}=0.68, \mathrm{p}=0.001)$.

\section{Discussion}

In the present study, we found significant differences in the levels of various stem cell markers (CD133, SOX2, OCT4 and TWIST1) correlated with colon cancer behavior. There was a reduction in expression when the tumors were responsive to the chemotherapy, and in contast, increased levels were noted when there was no response to treatment. Thus, the expression of these markers predicted the behavior of the tumor.

Research has focused on the study of tumor stem cells, in particular, in regards to the expression of markers to facilitate the identification of putative stem cells in tumors. Most of them are developed in tissue from a surgical tumor resection using a histological study. According to the cancer stem cell theory, CSCs are initiators of metastasis. Thus, our objective was to isolate them in peripheral blood. This procedure was used previously, and it affords the advantages of simplicity and easy accessibility to patient samples, without biopsy. 
RT-PCR has been widely used for the detection of CSCs in the peripheral blood for a variety of cancer types. RT-PCR is extremely sensitive, less subjective and can be automated, but its specificity depends on the cancer marker used (32-34).

In the present study, expression of c-Kit and CD133 was found in primary tumors, and CD133 expression was consistently higher in all colon cancer cases as compared to the controls, thus ruling out the possibility that $\mathrm{CD}_{133^{+}}$cells came from bone marrow. This difference in expression could be used as a biomarker, since the level in patients was almost 2 -fold when compared to that in the controls. The level was decreased in patients responsive to treatment, whereas in patients exhibiting disease progression an increase in levels was noted, due to an increase in the cell numbers in the tumor. There are many putative markers of cancer stem cells. Expression of these markers change depending on the specific tumor. Yet, at present, there is no doubt that CD133 is an effective stem cell marker for colon cancer. There are discrepancies concerning the pattern and frequency of CD133 expression in colon cancer. Horst et al (13) and Kojima et al (14) reported that CD133 expression is localized on glandular-luminal surface of colorectal cancer. However, Chun-Yan et al (8) showed that CD133 expression is located not only on the apical membrane but also on the basal surface of tumor cells, both in the invasive front. Nevertheless, we must consider the heterogeneous pathways and that CD133 expression levels are variable depending on the study.

Our data demonstrated that levels of SOX2 and OCT4 were highly correlated in patients and their trend was similar to CD133. It is well known that SOX2 and OCT4 are associated with the maintenance of stem cells, and play a critical role in the determination, differentiation and proliferation of stem cells, explicating the correlation between them and CD133. This positive correlation means that there is an increase in the number of stem cells and all the mechanisms implicated in proliferation and maintenance are activated (28,29-35).

The levels of the three markers, CD133, SOX2 and OCT4, were higher than in the controls in all cases, indicating that this was not due to maintenance of the physiological stem cells and is in relation with circulating cancer stem cells. Furthermore, their levels were reduced in those patients who were responsive to treatment, and increased in patients who were not responsive. We also observed a statistically significant correlation of the three markers at all sample collection points. This was in accordance with Saigusa et al (36) who reported that expression of the 3 genes (CD133, SOX2 and OCT4) was identified in residual CSCs and was associated with distal recurrences and poor disease-free survival which is linked to cancer cells that survive radical treatment, in support of the theory of metastasis due to stem cells. Our data indicate that CD133, OCT4, SOX2 had a predictive value for colon cancer behavior, and the expression may be useful for predicting the evolution of the treatment process.

Similar results were found for TWIST1 which regulates EMT in vertebrates. TWIST1 is highly expressed in several tumor types and its upregulation is considered an unfavorable prognostic factor in colorectal cancer. We demonstrated that the levels of TWIST1 exhibited the same trend as that of the other markers, but compared to all the markers, it indicated a favorable response to treatment. This suggests that it is a good indicator of disease progression and response to therapy.
Furthermore, TWIST2 was highly correlated with the other markers. This indicated that EMT occurs in stem cells in order to facilitate circulation in the peripheral blood, which is a protective mechanism for transit to distant organs. OCT4 and SOX2 are required for their maintenance, and once the cells reached a distant site they again began to show more specific markers. TWIST1 had predictive value for colon cancer behavior, and its correlation with OCT4 and the other markers may be useful for predicting the evolution of the treatment process (37).

The gene c-Kit, or stem cell factor, was the only marker exhibiting a different trend. In patients prior to treatment and after one dose of chemotherapy, the levels of c-Kit were lower in the patients when compared with that in the controls, while after the fourth dose of treatment the levels increased. This suggests that expression of c-Kit in colon tumors is rare; thus, it is not a marker of choice in the colon. These findings are in accordance with Friederichs et al (38). Yet, the increased expression after several dose of chemotherapy can indicate a change in receptor expression pathway of stem cells, or an alteration in the tyrosine-kinase receptors.

In conclusion, expression of CD133, OCT4, SOX2 and TWIST1 had a predictive value for colon cancer behavior. Evaluation of the expression of these stem cell gene markers may be useful for predicting the evolution of the treatment process, and the relative easy access to samples facilitates this method. Moreover, the correlation between CD133 and TWIST1 expression may be associated with tumor recurrence and metastatic relapse. These findings open new lines of research for the development of techniques for the study of circulating cancer stem cells and have implications in the research of the progression of colon cancer and have predictive value in translational oncology.

\section{References}

1. Howlader N, Noone AM, Krapcho M, Neyman N, Aminou R, Waldron W, Altekruse SF, Kosary CL, Ruhl J, Tatalovich Z, Cho H, Mariotto A, Eisner MP, Lewis DR, Chen HS, Feuer EJ, Cronin KA and Edwards BK (eds): SEER Cancer Statistics Review, 1975-2008, National Cancer Institute. Bethesda, MD, 2011.

2. Cunningham D, Atkin W, Lenz HJ, Lynch HT, Minsky B, Nordlinger B and Starling N: Colorectal cancer. Lancet 375: 1030-1047, 2010

3. Liao Y, Hu X, Huanq X and He C: Quantitative analyses of CD133 expression facilitate researches on tumor stem cells. Biol Pharm Bull 33: 738-742, 2010.

4. Boman BM, Wicha MS, Fields JZ, et al: Symmetric division of cancer stem cells - a key mechanism in tumor growth that should be targeted in future therapeutic approaches. Clin Pharmacol Ther 81: 893-898, 2007.

5. Boman, BM, Fields JZ, Cavanaugh KL, Guetter A and Runquist OA: How dysregulated colonic crypt dynamics cause stem cell overpopulation and initiate colon cancer. Cancer Res 68: 3304-3313, 2008

6. Morrison SJ and Kimble J: Asymmetric and symmetric stem-cell divisions in development and cancer. Nature 441: 1068-1074, 2006.

7. Johnston MD, Edwards CM, Bodmer WF, Maini PK and Chapman SJ: Mathematical modeling of cell population dynamics in the colonic crypt and in colorectal cancer. Proc Natl Acad Sci USA 104: 4008-4013, 2007.

8. Li CY, Li BX, Liang Y, et al: Higher percentage of $\mathrm{CD} 133^{+}$cells is associated with poor prognosis in colon carcinoma patients with stage IIIB. J Transl Med 7: 56, 2009. 
9. Huff CA, Matsui WH, Smith DB and Jones RJ: Strategies to eliminate cancer stem cells: clinical implications. Eur J Cancer 42: 1293-1297, 2006.

10. Klonisch T, Wiechec E, Hombach-Klonisch S, Ande SR, Wesselborg S, Schulze-Osthoff K and Los M: Cancer stem cell markers in common cancers - therapeutic implications. Trends Mol Med 14: 450-460, 2008.

11. Shmelkov SV, Butler JM, Hooper AT, et al: CD133 expression is not restricted to stem cells, and both $\mathrm{CD}_{133^{+}}$and $\mathrm{CD} 133$ metastatic colon cancer cells initiate tumors. J Clin Invest 118: 2111-2120, 2008.

12. Saigusa S, Tanaka K, Toiyama Y, et al: Clinical significance of CD133 and hypoxia inducible factor- $1 \alpha$ gene expression in rectal cancer after preoperative chemoradiotherapy. Clin Oncol (R Coll Radiol) 23: 323-332, 2011.

13. Horst D, Kriegl L, Engel J, Kirchner T and Junq A: Prognostic significance of the cancer stem cell markers CD133, CD44, and CD166 in colorectal cancer. Cancer Invest 27: 844-850, 2009.

14. Kojima M, Ishii G, Atsumi N, Fujii S, Saito N and Ochiai A: Immunohistochemical detection of CD133 expression in colorectal cancer: a clinicopathological study. Cancer Sci 99: 1578-1583, 2008.

15. Al-Hajj M, Wicha MS, Benito-Hernandez A, Morrison SJ and Clarke MF: Prospective identification of tumorigenic breast cancer cells. Proc Natl Acad Sci USA 100: 3983-3988, 2003.

16. Singh SK, Hawkins C, Clarke ID, et al: Identification of human brain tumour initiating cells. Nature 432: 396-401, 2004.

17. Boman BM and Wicha MS: Cancer stem cells: a step toward the cure. J Clin Oncol 26: 2795-2799, 2008.

18. Yin AH, Miraglia S, Zanjani ED, et al: AC133, a novel marker for human hematopoietic stem and progenitor cells. Blood 90: 5002-5012, 1997.

19. Zeki SS, Graham TA and Wright NA: Stem cells and their implications for colorectal cancer. Nat Rev Gastroenterol Hepatol 8: 90-100, 2011.

20. Lau CK, Yang ZF and Fan ST: Role of stem cells in normal liver and cancer. Anticancer Agents Med Chem 11: 522-528, 2011.

21. Monzani E, Facchetti F, Galmozzi E, et al: Melanoma contains CD133 and ABCG2 positive cells with enhanced tumourigenic potential. Eur J Cancer 43: 935-946, 2007.

22. Eramo A, Haas TL and De Maria R: Lung cancer stem cells: tools and targets to fight lung cancer. Oncogene 29: 4625-4635, 2010.

23. Ma S, Chan KW, Hu L, et al: Identification and characterization of tumorigenic liver cancer stem/progenitor cells. Gastroenterology 132: 2542-2556, 2007.

24. Maitland NJ and Collins AT: Cancer stem cells - A therapeutic target?. Curr Opin Mol Ther 12: 662-673, 2010.

25. Edling CE and Hallberg B: c-Kit - a hematopoietic cell essential receptor tyrosine kinase. Int J Biochem Cell Biol 39: 1995-1998, 2007.

26. Niwa H, Miyazaki J and Smith AG: Quantitative expression of Oct-3/4 defines differentiation, dedifferentiation or self-renewal of ES cells. Nat Genet 24: 372-376, 2000.
27. Looijenga LH, Stoop H, de Leeuw HP, et al: POU5F1 (OCT3/4) identifies cells with pluripotent potential in human germ cell tumors. Cancer Res 63: 2244-2250, 2003.

28. Tsukamoto T, Mizoshita T, Mihara M, et al: Sox 2 expression in human stomach adenocarcinomas with gastric and gastric-andintestinal-mixed phenotypes. Histopathology 46: 649-658, 2005.

29. Jia X, Li X, Xu Y, et al: SOX2 promotes tumorigenesis and increases the anti-apoptotic property of human prostate cancer cells. J Mol Cell Biol 3: 230-238, 2011.

30. Casas E, Kim J, Bendesky A, Ohno-Machado L, Wolfe CJ and Yang J: Snail2 is an essential mediator of Twist1-induced epithelial mesenchymal transition and metastasis. Cancer Res 71: 245-254, 2011.

31. Aktas B, Tewes M, Fehm T, Hausch S, Kimmig R and KasimirBauer S: Stem cell and epithelial-mesenchymal transition markers are frequently overexpressed in circulating tumor cells of metastatic breast cancer patients. Breast Cancer Res 11: R46, 2009.

32. Xi L, Nicastri DG, El-Hefnawy T, Hughes SJ, Luketich JD and Godfrey TE: Optimal markers for real-time quantitative reverse transcription PCR detection of circulating tumor cells from melanoma, breast, colon, esophageal, head and neck, and lung cancers. Clin Chem 53: 1206-1215, 2007.

33. Iinuma $\mathrm{H}$, Watanabe $\mathrm{T}$, Mimori $\mathrm{K}$, et al: Clinical significance of circulating tumor cells, including cancer stem-like cells, in peripheral blood for recurrence and prognosis in patients with Dukes' stage B and C colorectal cancer. J Clin Oncol 29: 1547-1555, 2011.

34. Sher YP, Shih JY, Yanq PC, et al: Prognosis of non-small cell lung cancer patients by detecting circulating cancer cells in the peripheral blood with multiple marker genes. Clin Cancer Res 11: 173-179, 2005.

35. Ong CW, Kim LG, Kong HH, et al: CD133 expression predicts for non-response to chemotherapy in colorectal cancer. Mod Pathol 23: 450-457, 2010.

36. Saigusa S, Tanaka K, Toiyama Y, et al: Correlation of CD133, OCT4, and SOX2 in rectal cancer and their association with distant recurrence after chemoradiotherapy. Ann Surg Oncol 16: 3488-3498, 2009.

37. Li QQ, Xu JD, Wanq WJ, et al: Twist1-mediated adriamycininduced epithelial-mesenchymal transition relates to multidrug resistance and invasive potential in breast cancer cells. Clin Cancer Res 15: 2657-2665, 2009.

38. Friederichs J, von Weyhern CW, Rosenberg R, et al: Immunohistochemical detection of receptor tyrosine kinases c-kit,EGF-R, and PDGF-R in colorectal adenocarcinomas. Langenbecks Arch Surg 395: 373-379, 2010.

39. Costelloe CM, Chuang HH, Madewell JE and Ueno NT: Cancer response criteria and bone metastases: RECIST 1.1, MDA and PERCIST. J Cancer 28: 80-92, 2010.

40. Sobin LH, Gospodarowicz MK and Wittekind C (eds): TNM Classification of Malignant Tumours. 7th edition. WileyBlackwell, New York, 2009. 